\title{
Effective interactions in the binary metallic systems
}

\author{
S.P.Koval' ${ }^{1}$, V.B.Solovyan ${ }^{2}$ \\ 1 The Ivan Franko National University of Lviv, 8 Kyrylo and Mephodii Str., \\ Lviv, 79005, Ukraine \\ 2 Institute for Condensed Matter Physics of the National Academy of \\ Sciences of Ukraine, 1 Svientsitskii Str., 79011, Lviv, Ukraine
}

Received February 24, 2004

The basic approach in the microscopic theory of binary metal systems has been developed. The electron-ion model Hamiltonian with nonlocal manyparticle interactions was obtained using the statistical operator averaging for the electron-nuclear model over the localized electron states. The role of orthogonalization and exchange effects on the formation of electron-ion interactions is investigated. The transition to the ion metal model with effective manyparticle interactions was performed.

Key words: basic approach, electron-nuclei model, electron-ion model, ion model, manyparticle interactions

PACS: $71.55 . A k$

The investigation of manyparticle effective interactions in the binary metallic systems is very interesting from different viewpoints. In particular, it is important in interpreting thermodynamic and structural characteristics of alloys and melts, in describing the quantum states of impurities in metals, structure phase transitions etc.

The basic approach, developed in papers [1,2], makes it possible to perform precise calculations without employing the model isights or any adjusted parameters. It gives the advantage of coupling the analytical and numerical analysis which becomes very substantial for the subsequent employment of the results produced.

In accordance with the basic approach let us consider the electron-nuclei model for the binary metallic system which consists of two types $(c=a, b)$ of nuclei $\left(N_{c}\right.$ is the number of nuclei of $c$ type with the charge $\left.Q_{c} \cdot e\right)$ and $N_{e}=\sum_{c} N_{c} Q_{c}$ electrons, $-e$ is the electron charge.

To describe the electron subsystem we use the compound basis of one particle wave functions

$$
\left\{\Psi_{\sigma}\right\}=\left\{\Psi_{l_{a}}\right\} \oplus\left\{\Psi_{l_{b}}\right\} \oplus\left\{\Psi_{\mathbf{k}}\right\}
$$


which consists of two orthogonal subspaces - localized $\left\{\Psi_{l_{c}}(\mathbf{r})\right\}(c=a, b)$ and delocalized functions $\left\{\Psi_{\mathbf{k}}(\mathbf{r})\right\}$. The functions of localized electron states are formed from the atomic orbitals $\varphi_{\mu_{c}, i_{c}}(\mathbf{r}) \equiv \varphi_{\mu_{c}}\left(\mathbf{r}-\mathbf{R}_{i_{c}}\right)$ according to the Bogoliubov method [3], for example

$$
\begin{aligned}
\Psi_{\lambda_{a}, j_{a}}(\mathbf{r})= & \varphi_{\lambda_{a}, j_{a}}(\mathbf{r})-\frac{1}{2} \sum_{\mu_{a}} \sum_{i_{a}\left(\neq j_{a}\right)}\left(\varphi_{\mu_{a}, i_{a}}, \varphi_{\lambda_{a}, j_{a}}\right) \varphi_{\mu_{a}, i_{a}}(\mathbf{r}) \\
& -\frac{1}{2} \sum_{\mu_{b}} \sum_{i_{b}}\left(\varphi_{\mu_{b}, i_{b}}, \varphi_{\lambda_{a}, j_{a}}\right) \varphi_{\mu_{b}, i_{b}}(\mathbf{r})+\cdots
\end{aligned}
$$

where $\lambda_{a}, \mu_{a}$ are the quantum states numbers of localized electrons that form the ionic cores, and $\mathbf{R}_{j_{a}}, \mathbf{R}_{i a}$ are nuclei coordinates. The subspace of $\Psi_{\mathbf{k}}(\mathbf{r})$-functions can be constructed using different methods, for instance with the help of the technique described in paper [4] and grounded on the plane wave basis $\left\{\varphi_{\mathbf{k}}\right\}$.

Let us calculate the partition function of the above described model over the electron variables in grand canonical ensemble

$$
Z_{\mu}=S p_{e}\left\{\exp \left[-\beta\left(\hat{H}-\mu \hat{N}_{e}\right)\right]\right\},
$$

which plays a role of the effective statistical operator for nuclei (ionic) subsystem. The Hamiltonian of an initial model in the second quantization representation takes the form

$$
\begin{aligned}
\hat{H}= & \hat{H}_{n}(\mathbf{R})+\sum_{\sigma_{1}, \sigma_{2}} \sum_{s} \epsilon_{\sigma_{1}, \sigma_{2}} a_{\sigma_{1}, s}^{+} a_{\sigma_{2}, s} \\
& +\frac{1}{2} \sum_{\sigma_{1}, \ldots, \sigma_{4}} \sum_{s_{1}, s_{2}} V_{\sigma_{1}, \sigma_{2}, \sigma_{3}, \sigma_{4}} a_{\sigma_{1}, s_{1}}^{+} a_{\sigma_{2}, s_{2}}^{+} a_{\sigma_{3}, s_{2}} a_{\sigma_{4}, s_{1}},
\end{aligned}
$$

where the component $\hat{H}_{n}(\mathbf{R})$ is the operator sum of the nuclei kinetic energy and their mutual Coulomb interaction. The second term in the right part of (4) presents the electrons' kinetic energy and attraction with nuclei, the third term presents electron-electron interaction. Here $a_{\sigma, s}$ are Fermi operators, which correspond to $\Psi_{\sigma}(\mathbf{r})$-functions (with the spin projection $s$ ), $\{\sigma\}=\left\{\lambda_{a}, j_{a}\right\},\left\{\lambda_{b}, j_{b}\right\},\{\mathbf{k}\}^{\prime}$. The "stroke" means that some number of wave vectors, which is equal to the number of localized functions in $\left\{\Psi_{l_{a}}\right\},\left\{\Psi_{l_{b}}\right\}$ subspaces are excluded from $\mathbf{k}$-spectrum. Matrix elements in (4) are defined as follows:

$$
\begin{aligned}
\epsilon_{\sigma_{1}, \sigma_{2}} & =T_{\sigma_{1}, \sigma_{2}}+\sum_{c} \sum_{j_{c}=1}^{N_{c}} v_{\sigma_{1}, \sigma_{2}}^{j_{c}}, \\
v_{\sigma_{1}, \sigma_{2}}^{j_{c}} & =-\frac{Q_{c}}{V} \sum_{\mathbf{q}} V_{q} R_{\sigma_{1}, \sigma_{2}}(\mathbf{q}) \exp \left(-\mathbf{i q} \mathbf{R}_{j_{c}}\right), \\
V_{\sigma_{1}, \sigma_{2}, \sigma_{3}, \sigma_{4}} & =\frac{1}{V} \sum_{\mathbf{q} \neq 0} V_{q} R_{\sigma_{1}, \sigma_{4}}(\mathbf{q}) R_{\sigma_{2}, \sigma_{3}}(-\mathbf{q}), \\
T_{\sigma_{1}, \sigma_{2}} & =\left(\Psi_{\sigma_{1}}\left|-\hbar^{2} \nabla^{2} / 2 m\right| \Psi_{\sigma_{2}}\right), \\
R_{\sigma_{1}, \sigma_{2}}(\mathbf{q}) & =\left(\Psi_{\sigma_{1}}|\exp (\mathbf{i q r})| \Psi_{\sigma_{2}}\right), \quad V_{q}=\frac{4 e^{2}}{\pi} q^{2} .
\end{aligned}
$$


According to the papers [1,2], we will split the calculation of $Z_{e}(\mu)$ into two stages. In the first stage we will calculate the track of statistical operator over the localized electron states $\lambda_{c}(c=a, b)$. In the second one - over the collectivized electron states. After the first stage computation we obtain the effective statistical operator of the collectivized electron subsystem in the ionic field, which determines the Hamiltonian of electron-ion model on $\left\{\Psi_{\mathbf{k}}\right\}$-subspace:

$$
\begin{aligned}
& S p_{l_{c}} \quad\left\{\exp \left[-\beta\left(\hat{H}-\mu \hat{N}_{e}\right)\right]\right\}=\exp \left[-\beta\left(\hat{H}_{\mathrm{ef}}-\mu \hat{N}_{k}\right)\right] \\
& \hat{H}_{\mathrm{ef}}=\hat{H}_{i}(\mathbf{R})+\sum_{\mathbf{k}_{1}, \mathbf{k}_{2}}{ }^{\prime} \sum_{s} v_{2}\left(\mathbf{k}_{1}, \mathbf{k}_{2} \mid \mathbf{R}\right) a_{\mathbf{k}_{1}, s}^{+} a_{\mathbf{k}_{2}, s} \\
& \quad+\frac{1}{2} \sum_{\mathbf{k}_{1}, \mathbf{k}_{2}, \mathbf{k}_{3}, \mathbf{k}_{4}} \sum_{s_{1}, s_{2}} v_{4}\left(\mathbf{k}_{1}, \mathbf{k}_{2}, \mathbf{k}_{3}, \mathbf{k}_{4} \mid \mathbf{R}\right) a_{\mathbf{k}_{1}, s_{1}}^{+} a_{\mathbf{k}_{2}, s_{2}}^{+} a_{\mathbf{k}_{3}, s_{2}} a_{\mathbf{k}_{4}, s} \\
&+\cdots, \\
& \hat{N}_{k}= \sum_{\mathbf{k}, s}{ }^{\prime} a_{\mathbf{k}, s}^{+} a_{\mathbf{k}, s} .
\end{aligned}
$$

Here

$$
\begin{aligned}
\hat{H}_{i}(\mathbf{R})= & \sum_{c} N_{c} E_{c}^{0}+\sum_{c}\left[-\hbar^{2} /\left(2 M_{c}\right)\right] \sum_{j_{c}} \nabla_{j_{c}}^{2} \\
& +\frac{1}{2} \sum_{c_{1}, c_{2}} \sum_{j_{c_{1}}, j_{c_{2}}} V_{c_{1}, c_{2}}\left(\mathbf{R}_{j_{c_{1}}}-\mathbf{R}_{j_{c_{2}}}\right)
\end{aligned}
$$

is the Hamiltonian of isolated ion subsystem, $E_{c}^{0}$ is the energy of an isolated ion and $V_{c_{1}, c_{2}}\left(\mathbf{R}_{j_{c_{1}}}-\mathbf{R}_{j_{c_{2}}}\right)$ is the pair ion-ion potential in the Hartree-Fock approximation $\left(j_{c_{1}} \neq j_{c_{2}}\right.$ at $\left.c_{1}=c_{2}\right)$,

$$
\begin{aligned}
& V_{c_{1}, c_{2}}(\mathbf{R})=\frac{1}{V} \sum_{\mathbf{q}} V_{c_{1}, c_{2}}(\mathbf{q}) \exp (\mathbf{i q R}) \\
& V_{c_{1}, c_{2}}(\mathbf{q})=V_{q} Z_{c_{1}}(\mathbf{q}) Z_{c_{1}}(\mathbf{q})
\end{aligned}
$$

where

$$
Z_{c}(\mathbf{q})=Q_{c}-2 \sum_{\lambda_{c}} R_{\lambda_{c}, \lambda_{c}}(\mathbf{q})
$$

is the effective ion valency function, which at $q \rightarrow 0$ tends to its true value - the ion valency $Z_{c}$. The second term in $\hat{H}_{\text {ef }}$ describes the one particle electron-ion interaction and the third describes the two electron interaction in the ion field.

To simplify the second stage computations (the averaging over the conductive electron states ) we will turn from operators $a_{\mathbf{k}, s}$ to $C_{\mathbf{k}, s}$, which conforms with plane waves basis $\left\{\varphi_{\mathbf{k}}(\mathbf{r})\right\}=\left\{V^{-1 / 2} \exp (\mathbf{i k r})\right\}$ according to the expressions:

$$
a_{\mathbf{k}, s}=\sum_{\mathbf{q}}\left(\Psi_{\mathbf{k}}, \varphi_{\mathbf{q}}\right) C_{\mathbf{q}, s}
$$


This relationship, simultaneously with the completeness condition for $\left\{\Psi_{\sigma}\right\}$-basis, written in the momentum space

$$
\sum_{l}\left(\Psi_{l}, \varphi_{\mathbf{q}_{1}}\right)\left(\varphi_{\mathbf{q}_{2}}, \Psi_{l}\right)+\sum_{\mathbf{k}}^{\prime}\left(\Psi_{\mathbf{k}}, \varphi_{\mathbf{q}_{1}}\right)\left(\varphi_{\mathbf{q}_{2}}, \Psi_{\mathbf{k}}\right)=\delta_{\mathbf{q}_{1}, \mathbf{q}_{2}}
$$

permits to recalculate the Hamiltonian $\hat{H}_{\text {ef }}$ matrix elements. The sum calculation over the wave vectors $\mathbf{k}$ can be done in the general form without concrete definition of functions $\Psi_{\mathbf{k}}(\mathbf{r})$. As a result, the general form of Hamiltonian $\hat{H}_{\text {ef }}$ remains, but instead of matrix elements $T_{\sigma_{1}, \sigma_{2}}, R_{\sigma_{1}, \sigma_{2}}(\mathbf{q})$ (see (5)), calculated on the basic functions $\Psi_{\sigma}(\mathbf{r})$, we obtain matrix elements $T_{\sigma_{1}, \sigma_{2}}^{0}, R_{\sigma_{1}, \sigma_{2}}^{0}(\mathbf{q})$ which are calculated on the functions of $O P W$-system:

$$
\left\{\Psi_{l_{a}}(\mathbf{r})\right\} \oplus\left\{\Psi_{l_{b}}(\mathbf{r})\right\} \oplus\left\{\chi_{\mathbf{k}}(\mathbf{r})\right\}
$$

where

$$
\chi_{\mathbf{k}}(\mathbf{r})=\varphi_{\mathbf{k}}(\mathbf{r})-\sum_{c} \sum_{l_{c}}\left(\Psi_{l_{c}}, \varphi_{\mathbf{k}}\right) \Psi_{l_{c}}(\mathbf{r})
$$

- orthogonalized plane wave. Thus, we obtained $\{\sigma\}=\left\{l_{a}\right\} \oplus\left\{l_{b}\right\} \oplus\{\mathbf{k}\}$ without any restrictions for the wave vector $\mathbf{k}$.

Further, we extract from operator $\hat{H}_{\text {ef }}$ the electron liquid Hamiltonian $\hat{H}_{\text {EL }}$ and the electron-ion interaction operator $\hat{V}_{\mathrm{ei}}$ :

$$
\begin{aligned}
\hat{H}_{\mathrm{ef}}= & \hat{H}_{i}(\mathbf{R})+\hat{H}_{\mathrm{EL}}+\hat{V}_{\mathrm{ei}} \\
\hat{H}_{\mathrm{EL}}= & \sum_{\mathbf{k}, s} \varepsilon_{\mathbf{k}} a_{\mathbf{k}, s}^{+} a_{\mathbf{k}, s}+\frac{1}{2 V} \sum_{\mathbf{q}} V_{q} \sum_{\mathbf{k}_{1}, \mathbf{k}_{2}} \sum_{s_{1}, s_{2}} a_{\mathbf{k}_{1}+\mathbf{q}, s_{1}}^{+} a_{\mathbf{k}_{2}-\mathbf{q}, s_{2}}^{+} a_{\mathbf{k}_{2}, s_{2}} a_{\mathbf{k}_{1}, s_{1}} \\
\hat{V}_{\mathrm{ei}}= & \sum_{\mathbf{k}_{1}, \mathbf{k}_{2}} \sum_{s} A_{2}\left(\mathbf{k}_{1}, \mathbf{k}_{2} \mid \mathbf{R}\right) a_{\mathbf{k}_{1}, s}^{+} a_{\mathbf{k}_{2}, s} \\
& +\frac{1}{2} \sum_{\mathbf{k}_{1}, \ldots, \mathbf{k}_{4}} \sum_{s_{1}, s_{2}} A_{4}\left(\mathbf{k}_{1}, \mathbf{k}_{2}, \mathbf{k}_{3}, \mathbf{k}_{4} \mid \mathbf{R}\right) a_{\mathbf{k}_{1}, s_{1}}^{+} a_{\mathbf{k}_{2}, s_{2}}^{+} a_{\mathbf{k}_{3}, s_{2}} a_{\mathbf{k}_{4}, s_{1}}+\cdots
\end{aligned}
$$

where $\varepsilon_{\mathbf{k}}=\hbar^{2} k^{2} / 2 m$. We present here the main terms of matrix elements $A_{2}\left(\mathbf{k}_{1}, \mathbf{k}_{2} \mid \mathbf{R}\right), A_{4}\left(\mathbf{k}_{1}, \mathbf{k}_{2}, \mathbf{k}_{3}, \mathbf{k}_{4} \mid \mathbf{R}\right)$, disregarding the components caused by gibridization effects between localized and delocalized electrons :

$$
\begin{aligned}
A_{2}\left(\mathbf{k}_{1}, \mathbf{k}_{2} \mid \mathbf{R}\right)= & T_{\mathbf{k}_{1}, \mathbf{k}_{2}}^{0}-\varepsilon_{\mathbf{k}_{1}} \delta_{\mathbf{k}_{1}, \mathbf{k}_{2}}-\frac{1}{V} \sum_{c} Q_{c} \sum_{j_{c}} R_{\mathbf{k}_{1}, \mathbf{k}_{2}}^{0}(\mathbf{q}) \exp \left(-\mathrm{iq} \mathbf{R}_{j_{c}}\right) \\
& +\frac{1}{V} \sum_{c} \sum_{l_{c}, s} \sum_{\mathbf{q} \neq 0} V_{q}\left\{R_{\mathbf{k}_{1}, \mathbf{k}_{2}}^{0}(\mathbf{q}) R_{l_{c}, l_{c}}^{0}(-\mathbf{q})-\frac{1}{2} R_{\mathbf{k}_{1}, l_{c}}^{0}(\mathbf{q}) R_{l_{c}, \mathbf{k}_{2}}^{0}(-\mathbf{q})\right\} \\
& +A_{4}\left(\mathbf{k}_{1}, \mathbf{k}_{2}, \mathbf{k}_{3}, \mathbf{k}_{4} \mid \mathbf{R}\right)= \\
= & \frac{1}{V} \sum_{\mathbf{q} \neq 0} V_{q}\left\{R_{\mathbf{k}_{1}, \mathbf{k}_{4}}^{0}(\mathbf{q}) R_{\mathbf{k}_{2}, \mathbf{k}_{3}}^{0}(-\mathbf{q})-\delta_{\mathbf{k}_{1}, \mathbf{k}_{4}+\mathbf{q}} \delta_{\mathbf{k}_{2}, \mathbf{k}_{3}-\mathbf{q}}\right\} .
\end{aligned}
$$

We pass on to the second stage of computation, i.e., the partition function calculation over the collectivized electron states. As in the reference system we use the 
electron liquid model with Hamiltonian $\hat{H}_{\mathrm{EL}}$. We will take into account the electronion interactions in terms of perturbation theory method. As one can see from (2), (14), the operator $\hat{V}_{\text {ei }}$ describes nonlocal one- and two-electron interactions, which have manyparticle character relatively to ions.

In this paper, in order to avoid inconveniences, we restrict ourselves to a simple metal case, neglecting the atomic function $\varphi_{\mu_{a}}\left(\mathbf{r}-\mathbf{R}_{j_{a}}\right)$ overlap integrals. To simplify the high order diagram calculation for perturbation theory series we will restrict ourselves to the main terms in $A_{2}\left(\mathbf{k}_{1}, \mathbf{k}_{2} \mid \mathbf{R}\right)$ and $A_{4}\left(\mathbf{k}_{1}, \mathbf{k}_{2}, \mathbf{k}_{3}, \mathbf{k}_{4} \mid \mathbf{R}\right)$, which are additive over ion partial structure factors

$$
S_{q}^{c}=\sum_{j_{c}=1}^{N_{c}} \exp \left(\mathbf{i q} \mathbf{R}_{j_{c}}\right) .
$$

But in the first order of perturbation theory series we will also take into account the terms proportional to the product of two partial structure factors.

The statistical operator track for the electron-ion model over the electron variables defines the statistical operator for the ion model of the metal with effective manyparticle interactions:

$$
\begin{aligned}
\exp \left\{\beta \mu^{*} N_{e}\right\} \cdot S p_{e}(\exp & \left.\left\{-\beta\left[\hat{H}-\mu^{*} \hat{N}_{e}\right]\right\}\right)= \\
= & \exp \left\{-\beta\left[F_{\mathrm{EL}}+\sum_{c} N_{c} \omega_{c}+\hat{H}_{\mathrm{ef}}(\mathbf{R})\right]\right\}
\end{aligned}
$$

where $\mu^{*} \equiv \mu^{*}(\beta, N / V)$ is the electron chemical potential, $F_{\mathrm{EL}}$ is free energy for electron liquid model, $\omega_{c}$ is the single ion energy in the electron liquid surroundings in the pair correlation approximation relatively to the reference system, $\hat{H}_{\text {ef }}(\mathbf{R})$ is the ion subsystem Hamiltonian where the interionic energy is written in terms of structure factors:

$$
\begin{aligned}
\hat{H}_{\mathrm{ef}}(\mathbf{R})= & -\sum_{c} \sum_{j_{c}}\left[\frac{\hbar^{2}}{2 M_{c}} \nabla_{j_{c}}^{2}\right] \\
& +\frac{1}{2 V} \sum_{c_{1}, c_{2}} \sum_{\mathbf{q} \neq \mathbf{0}} V_{c_{1}, c_{2}}^{(2)}(\mathbf{q})\left[S_{\mathbf{q}}^{c_{1}} S_{-\mathbf{q}}^{c_{2}}-\delta_{c_{1}, c_{2}} N_{c_{1}}\right] \\
& +\sum_{n \geq 3} \frac{(-1)^{n-1}}{n ! V^{n-1}} \sum_{c_{1}, \ldots, c_{n}} \sum_{\mathbf{q}_{1}, \ldots, \mathbf{q}_{n}} V_{c_{1}, \ldots, c_{n}}^{(n)}\left(\mathbf{q}_{1}, \ldots, \mathbf{q}_{n}\right) S_{\mathbf{q}_{1}}^{c_{1}} \cdots S_{\mathbf{q}_{n}}^{c_{n}} \delta_{\mathbf{q}_{1}+\cdots+\mathbf{q}_{n}, 0}
\end{aligned}
$$

Here, we obtain the following expressions for the interionic potentials:

$$
\begin{aligned}
V_{c_{1}, c_{2}}^{(2)}(\mathbf{q})=V_{q} Z_{c_{1}}(\mathbf{q}) & Z_{c_{2}}(-\mathbf{q})-\frac{1}{V} \sum_{\mathbf{k}} a_{2}^{c_{1}}(\mathbf{k}+\mathbf{q}, \mathbf{k}) a_{2}^{c_{2}}(\mathbf{k}, \mathbf{k}+\mathbf{q}) \mu_{2}(\mathbf{q},-\mathbf{q} \mid \mathbf{k}) \\
V_{c_{1} \ldots c_{n}}^{(n)}\left(\mathbf{q}_{1}, \ldots, \mathbf{q}_{n}\right)= & \frac{1}{V} \sum_{\mathbf{k}} \mu_{n}\left(\mathbf{q}_{1}, \ldots, \mathbf{q}_{n} \mid \mathbf{k}\right) a_{2}^{c_{1}}\left(\mathbf{k}, \mathbf{k}-\mathbf{q}_{1}\right) a_{2}^{c_{2}}\left(\mathbf{k}-\mathbf{q}_{1}, \mathbf{k}-\mathbf{q}_{1}-\mathbf{q}_{2}\right) \\
& \times \cdots a_{2}^{c_{n}}\left(\mathbf{k}-\mathbf{q}_{1}-\cdots-\mathbf{q}_{n-1}, \mathbf{k}-\mathbf{q}_{1}-\cdots-\mathbf{q}_{n}\right)
\end{aligned}
$$


where $\mu_{n}\left(\mathbf{q}_{1}, \ldots, \mathbf{q}_{n} \mid \mathbf{k}\right)-\mathbf{k}$ is the component $n$-particle static semi-invariant correlation function of the reference system [5]. Particulary, in the local field approximation

$$
\mu_{2}(\mathbf{q},-\mathbf{q} \mid \mathbf{k})=\mu_{2}^{0}(\mathbf{q},-\mathbf{q} \mid \mathbf{k})\left\{1+V_{q} V^{-1} \mu_{2}^{0}(\mathbf{q},-\mathbf{q})\left[1-G_{q}\right]\right\}^{-1}
$$

where $G_{q}$ is static local field correction function and

$$
\mu_{2}^{0}(\mathbf{q},-\mathbf{q})=\sum_{\mathbf{k}} \mu_{2}^{0}(\mathbf{q},-\mathbf{q} \mid \mathbf{k})
$$

is the pair correlation function of noninteracting electron subsystem. The effective ion valence function is determined by expressions:

$$
\begin{aligned}
& Z_{c}(\mathbf{q})=Q_{e}-2 \sum_{\lambda_{c}} R_{\lambda_{c} \lambda_{c}}(\mathbf{q})-\frac{1}{V} \sum_{\mathbf{k}, s} n_{\mathbf{k}, s} P_{\mathbf{k}, \mathbf{k}}^{c}(\mathbf{q}) \\
& P_{\mathbf{k}, \mathbf{k}}^{c}(\mathbf{q})=\sum_{\lambda_{c}}\left\{\sum_{\mu_{c}} R_{\lambda_{c}, \mu_{c}}(\mathbf{q}) \varphi_{\lambda_{c}}(\mathbf{k}) \varphi_{\mu_{c}}^{*}(\mathbf{k})\right. \\
& \\
&\left.\quad-\varphi_{\lambda_{c}}(\mathbf{k}-\mathbf{q}) \varphi_{\lambda_{c}}^{*}(\mathbf{k})-\varphi_{\lambda_{c}}(\mathbf{k}) \varphi_{\lambda_{c}}^{*}(\mathbf{k}+\mathbf{q}) \sum_{\mu_{c}}\right\}
\end{aligned}
$$

The contributions to effective interionic potentials which appear in the second and higher orders in perturbation theory series are obtained in the following approximation for $\hat{V}_{\mathrm{ei}}$ :

$$
\hat{V}_{\mathrm{ei}} \Longrightarrow \hat{V}_{\mathrm{ei}}^{(0)} \equiv \sum_{c} \frac{1}{V} \sum_{\mathbf{q} \neq 0} S_{-\mathbf{q}} \sum_{\mathbf{k}} a_{2}(\mathbf{k}+\mathbf{q}, \mathbf{k}) C_{\mathbf{k}+\mathbf{q}, s}^{+} C_{\mathbf{k}, s} .
$$

Here, the main term of two-electron interaction is taken in Hartree-Fock approximation.

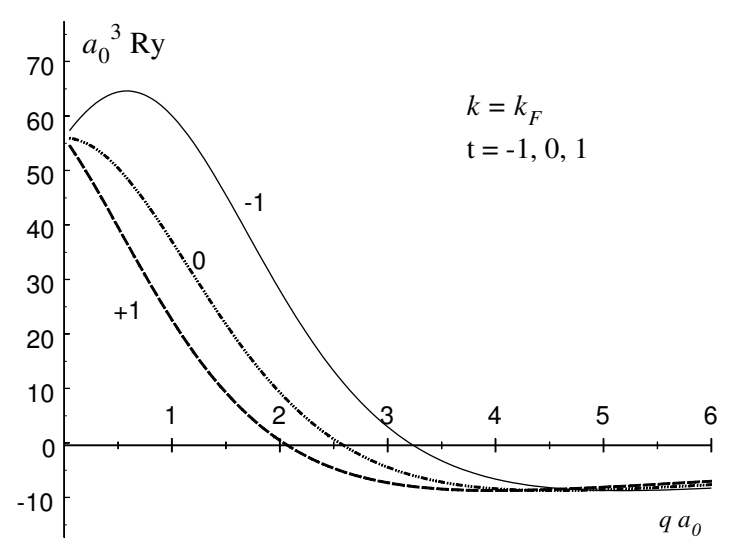

Figure 1. Depending of the kinetic energy contribution on wave vector $q\left(k=k_{\mathrm{F}}\right.$, $t=-1,0,1)$. 
In accordance with (18), $n$-particle interionic potentials are of the screening character, particularly $V_{c_{1}, c_{2}}^{(2)}(\mathbf{q})$ have the following asymptotics:

$$
\begin{aligned}
& \lim _{q \rightarrow 0}\left(V_{c_{1}, c_{2}}^{(2)}(\mathbf{q})\right)=V_{q} Z_{c_{1}}(\mathbf{q}) Z_{c_{2}}(\mathbf{q}) \frac{1-L(q) G(q)}{1+L(q)[1-G(q)]} \\
& \lim _{q \rightarrow \infty}\left(V_{c_{1}, c_{2}}^{(2)}(\mathbf{q})\right) \approx V_{q} Q_{c_{1}} Q_{c_{2}},
\end{aligned}
$$

where $L(q)=V_{q} V^{-1} \mu_{2}^{0}(q,-q)$. As we can see, the nonlocal matrix elements of electron-ion interaction, simultaneously with electron liquid model correlation functions, completely define the character of $n$-particle interionic potentials.

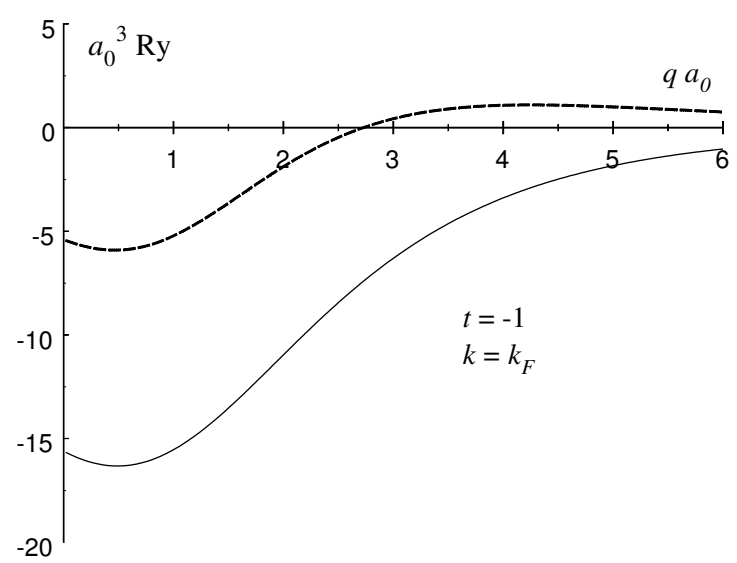

Figure 2. The exchange interaction between localized and conductive electrons in plane waves (solid curve) and ortogonalized plane waves (dashed curve).

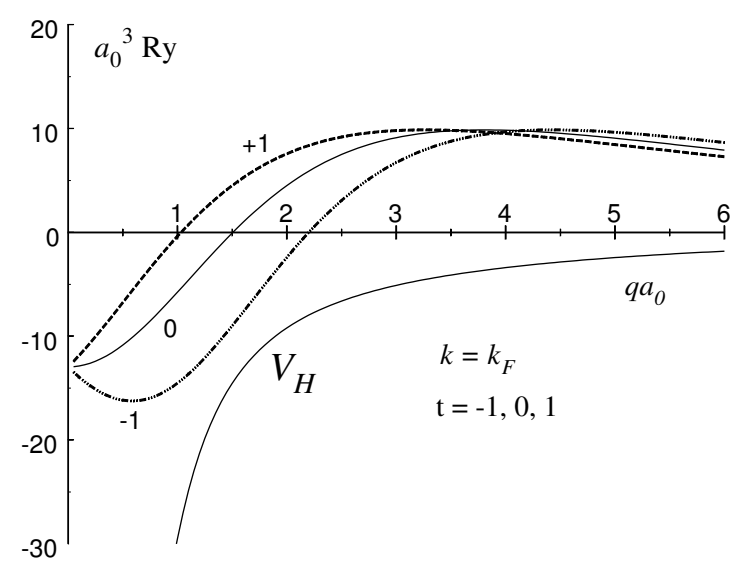

Figure 3. Dependence of direct electron-ion Coulomb interaction on wave vector $q$ at $k=k_{\mathrm{F}}$. The lovest curve coresponds to plane waves approsimation, other ones to ortogonalization corrections at $t=-1,0,1$.

For example, let us consider the properties of these matrix elements for the metallic lithium case, using a simple 1 s-function $\varphi_{1 s}(\mathbf{r})=\pi^{-1 / 2}\left(\alpha / a_{0}\right)^{3 / 2} \exp \left(-\alpha r / a_{0}\right)$, 


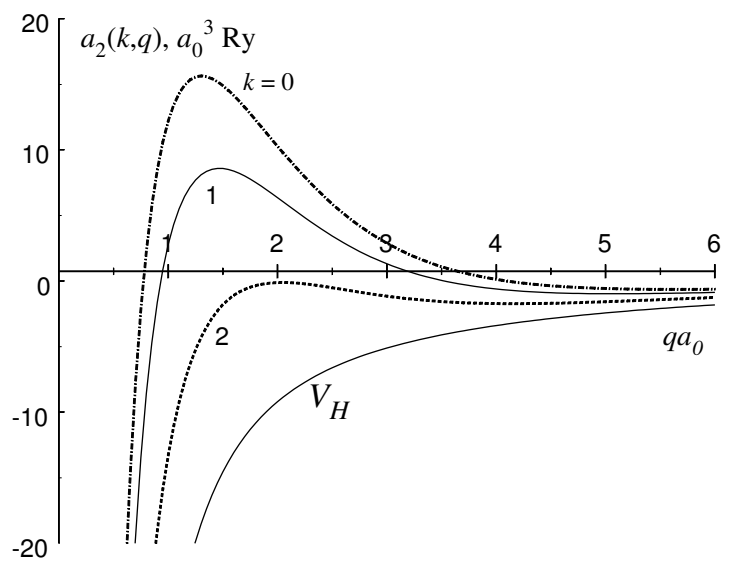

Figure 4. The total electron-ion formfactor $a_{2}(\mathbf{k}+\mathbf{q}, \mathbf{k})$, averaging over the angle between $\mathbf{k}$ and $\mathbf{q}$ at $k=0, k_{\mathrm{F}}, 2 k_{\mathrm{F}}$.

where $a_{0}$ is Bohr radius and $\alpha$ is a variational parameter (for an isolated ion $\mathrm{Li}^{+}$ parameter $\alpha$ takes the value 43/16). This form being sufficiently precise for $1 \mathrm{~s}$ electron description, makes it possible to perform the calculations for $a_{2}^{c}(\mathbf{k}+\mathbf{q}, \mathbf{k})$ in analytical form, which permits to perform the precise analysis of the obtained results.

Figures 1-3 show the main components of electron-ion formfactor $a_{2}^{c}(\mathbf{k}+\mathbf{q}, \mathbf{k})$ for the case $k=k_{\mathrm{F}}$ and a different mutual orientation of vectors $\mathbf{k}$ and $\mathbf{q}(t \equiv$ $\cos (\mathbf{k}, \mathbf{q})=-1,0,1)$. Figure 1 illustrates the contribution of electron kinetic energy change caused by orthogonalization effects. Figure 2 shows the contribution caused by exchange interaction between localized and conductive electrons in different approximations: dashed curve corresponds to plane wave approximation and the solid one corresponds to orthogonalized plane wave. Figure 3 illustrates the direct electron-ion Coulomb interaction. Lower curve corresponds to plane waves approximation, higher curves are orthogonalization corrections. Figure 4 illustrates the total

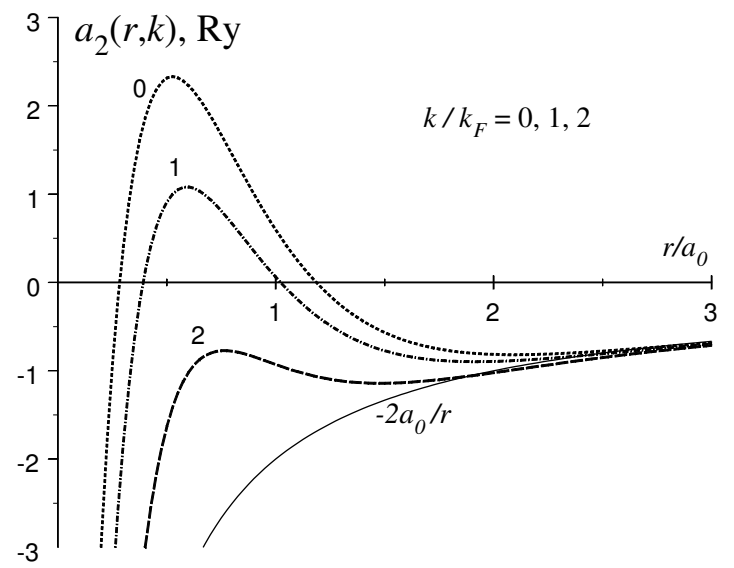

Figure 5. The function $a_{2}(r, k)$ for Li at $k=0, k_{\mathrm{F}}, 2 k_{\mathrm{F}}$. 
formfactor dependence over $k, q$ being the average over the angle between $\mathbf{k}$ and $\mathbf{q}$. All figures correspond to $\alpha=43 / 16$.

As one can see in figures $1-4$, the formfactor $a_{2}^{c}(\mathbf{k}+\mathbf{q}, \mathbf{k})$ speciality is its high nonlocality (dependence on modulus and orientation of vector $\mathbf{k}$ ), caused, firstly, by orthogonalization effects. The most important corrective term is the kinetic energy contribution (see figure 1). It generates the maximum on $a_{2}^{c}(\mathbf{k}+\mathbf{q}, \mathbf{k})$ curve (figure 4 ). The height of this maximum decreases with the electron kinetic energy increasing. We have calculated the Fourier transformation of formfactor $a_{2}^{c}(\mathbf{k}+\mathbf{q}, \mathbf{k})$, averaged over the $\mathbf{k}$ and $\mathbf{q}$ angle (figure 5 ) at $k=0, k_{\mathrm{F}}, 2 k_{\mathrm{F}}$. The $a_{2}(r, k)$ dependence has the Coulomb character $\left(r^{-1}\right)$ for small and for large values of $r\left(\lim _{r \rightarrow \infty}\left(a_{2}(r, k)\right)=\right.$ $\left.-Z^{*} e^{2} / r\right)$.

\section{References}

1. Vavrukh M.V., Muliava Y.V. // J. Phys. Stud., 1997, vol. 1, No. 2, p. 257 (in Ukrainian)

2. Vavrukh M.V., Yakibchuk P.M., Koval' S.P. // Metalofizyka i novitni tehnologii, 2000, vol. 22, No. 5 (in Russian)

3. Bogolyubov N.N. Izbrannye Trudy, vol. 2. Kyiv, Naukova dumka, 1970 (in Ukrainian)

4. Vavrukh M.V., Solovyan V.B. // Ukr. Jorn. Phys., 1991, vol. 36, No. 4, p. 630 (in Ukrainian)

5. Vavrukh M.V., Vavrukh N.M., Solovyan V.B. // Phys. Stat. Sol. (b), 1993, vol. 177, No. 2, p. 361 


\title{
Ефективні взаємодії у бінарних металічних системах
}

\author{
С.П.Коваль ${ }^{1}$, В.Б.Солов'ян ${ }^{2}$
}

1 Львівський національний університет імені Івана Франка, 79005 Львів, вул. Кирила і Мефодія, 8

2 Інститут фізики конденсованих систем НАН України, 79011 Львів, вул. Свєнціцького, 1

Отримано 24 лютого, 2004 р.

Розроблено базисний підхід у мікроскопічній теорії бінарних металічних систем. Шляхом статистичного засереднення статистичного оператора електрон-ядерної моделі за станами підсистеми локалізованих електронів побудовано гамільтоніан електрон-іонної моделі з нелокальними багаточастинковими взаємодіями. Досліджено роль ефектів ортогоналізації та обміну у формуванні електроніонних взаємодій. Здійснено перехід до іонної моделі з ефективними багаточастинковими взаємодіями.

Ключові слова: базисний підхід, електрон-ядерна модель, електрон-іонна модель, іонна модель, багаточастинкові взаємодії

PACS: $71.55 . A K$ 